\title{
Economía política de las propuestas de reforma de protección a la vejez en Colombia (2019-2020)
}

\section{Political economy of the proposals for the reform of Old Age Protection in Colombia (2019-2020)}

\section{José Silva Ruiz iD}

Escuela Superior de Administración Pública (ESAP)

josepsilvar@gmail.com

\begin{abstract}
Resumen
La economía política estudia la interconexión entre la economía y la política en un Estado que tiene como función esencial el desarrollo del bienestar de la sociedad y la asignación de recursos; en este caso, para asegurar una vejez digna de la población. Desde esta perspectiva se describen las propuestas de reforma bajo tres supuestos e instrumentos de análisis: 1) individualismo metodológico (intereses privados propios o de las instituciones que representan los actores); 2) elección racional (cálculos costo/beneficio) y, (3) la política como intercambio (beneficios y utilidades). El objetivo del trabajo consiste en un diagnóstico inicial del sistema pensional vigente, en reseñar las propuestas de reforma y la puesta a consideración de una propuesta del autor. El artículo es de reflexión sobre el sistema pensional y las propuestas de reforma, que implica una descripción sobre la normatividad y práctica, la interpretación de los principios o variables (cobertura, equidad, solidaridad y sostenibilidad) y de análisis crítico sobre las propuestas.
\end{abstract}

Palabras clave: Jubilación, cobertura, equidad, solidaridad, sostenibilidad fiscal.

JEL: J26, G23, D63, E21, H55.

\begin{abstract}
The political economy studies the interconnection between the economy and politics in a State whose essential function is the development of the welfare of society and the allocation of resources, in this case, to ensure an old age worthy of the population. From this perspective, the reform proposals are described under three assumptions and instruments of analysis: 1) methodological individualism (private interests of their own or of the institutions representing the actors); 2) rational choice (cost / benefit calculations) and, (3) the policy as an exchange (benefits and profits). The objective of the work consists in an initial diagnosis of the current pension system, in reviewing the reform proposals and the consideration of a proposal by the author. The article is a reflection on the pension system and the reform proposals, which implies a description of the regulations and practice, the interpretation of the principles or variables (coverage, equity, solidarity and sustainability) and a critical analysis of the proposals.
\end{abstract}

Keywords: Retirement, coverage, equity, solidarity, fiscal sustainability

JEL: H55, E21, H62, D63.

Articulo: Recibido el 9 de agosto de 2019 y aprobado 12 de noviembre de 2019.

\section{Cómo citar este artículo:}

Silvia Ruiz, J. (2019). Economía política de las propuestas de reforma de protección a la vejez en Colombia (20192020). Reflexión Política 21(43), pp. 90-106 doi: 10.29375/01240781.3679 


\section{Introducción}

La previsión social de manera formal se inicia en Colombia bajo la influencia del Estado de Bienestar, con la creación de dos instituciones oficiales, la Caja Nacional de Previsión Social, Cajanal (Ley 6 de 1945) para los empleados públicos, y el Instituto Colombiano de Seguros Sociales (ICSS, Ley 90 de 1946; posterior ISS, 1977), para cubrir a los trabajadores del sector público. En 1967 se establece un sistema para afiliados privados.

Con el surgimiento del modelo de "economía de mercado", la política de protección social y de servicios públicos cambió radicalmente en Colombia a partir de la reforma constitucional de 1991, y de normas de regulación como la Ley 100 de 1993 (sistema de seguridad social integral). La función del Estado "proveedor" dio paso a la del Estado "regulador", en el cual el sector público (descentralizado) y el sector privado funcionan como operadores de los servicios, y el Estado regula, controla y supervisa la provisión con el supuesto de proteger el bien común y el interés público.

La Constitución Política de 1991 en el artículo 48 establece que, "la seguridad social podrá ser prestada por entidades públicas o privadas, de conformidad con la ley". El gobierno de la época reestructuró el sistema pensional con la Ley 100 de 1993, la cual marcó el comienzo de la privatización de la seguridad social (salud y pensiones). El sistema pensional se materializó en dos instituciones: el Instituto de Seguros Sociales, ISS (sustituido a partir de octubre del año 2012 por la Administradora Colombiana de Pensiones, Colpensiones) y los Fondos de Pensiones Privados, AFP, con dos regímenes, el "Régimen Solidario de Prima Media", RPM, a cargo de la oficial Colpensiones y el "Régimen de Ahorro Individual con Solidaridad", RAIS, operado por las administradoras de fondos privados de pensiones y de cesantías, AFP.

Las reformas periódicas y sistemáticas al sistema pensional en Colombia (1993, 2003 y 2005) evidencian al igual que en los asuntos de economía pública que en últimas, la decisión es de tipo político, con gran incidencia de los intereses del sector privado (financiero) bajo el paraguas del modelo de "economía de mercado". En un sistema capitalista, el Estado, los gobiernos y la administración pública son proclives a los intereses del capital y en esencia a los del sector financiero.

El diagnóstico general señala los problemas del actual esquema, examinados desde la perspectiva de los cuatro principios de todo sistema pensional: cobertura, equidad, solidaridad y sostenibilidad. La cobertura de afiliados oscila entre el 34 y $36 \%$ de la población económicamente activa, el RPM cubre el $9,5 \%$ y el RAIS un $25,1 \%$. Los bajos niveles de cobertura tanto en porcentajes de cotizantes como del adulto mayor obedecen en buena parte a la incidencia de la informalidad laboral en Colombia.

En cuanto a la equidad, el sistema pensional es inequitativo y regresivo. El RPM es un sistema subsidiado, pero este lo reciben en alto porcentaje los quintiles 4 y 5. El Fondo de Solidaridad Pensional (FSP) se constituye en la fuente de financiación del Programa Colombia Mayor.

Buena parte de las predicciones de catástrofe del sistema ("bomba fiscal o social") se centran en los problemas de sostenibilidad. Las pensiones de los regímenes públicos (RPM + Especiales) no alcanzan a ser financiadas con las cotizaciones anuales de los trabajadores, y los gobiernos financian los faltantes con recursos del Presupuesto General de la Nación, PGN, que ha oscilado para los años 2018 y 2019 , en un monto cercano a los 40 billones, con un déficit de Colpensiones de 13 billones por año. Monto en todo caso inferior a los cerca de 65 billones que el PGN de 2019 debe destinar al servicio de la deuda pública nacional con el sector financiero nacional e internacional (28 billones en intereses y 37 billones a amortización de la deuda). La deuda del Gobierno Nacional Central, GNC, con los fondos privados es cercana a los $\$ 40$ billones.

La Asociación Colombiana de Administradoras de Fondos de Pensiones y Cesantías (Asofondos), con el apoyo de la Federación Internacional de Administradoras de Fondos de Pensiones (FIAP) y la Asociación de Instituciones Financieras (ANIF), han estado muy activas en Colombia en los tres últimos años, realizando o participando en congresos y seminarios, para mostrar las fallas del sistema pensional 
oficial, en particular la no sostenibilidad del "Régimen de Prima Media" (RPM), a cargo de Colpensiones, y en resaltar las bondades del "Régimen de Ahorro Privado con Solidaridad" (RAIS). Para la ANIF, se debe reducir el monto de la base pensional de $65 \%$ a $45 \%$, así como el monto máximo o tasa de reemplazo al 75\%; aumentar la edad de pensión a 65 años; igualar la edad de hombres y mujeres; y reforzar los esquemas no contributivos, los Beneficios Periódicos, BEPS, y Adulto Mayor.

Los organismos internacionales (Fondo Monetario Internacional [FMI], Banco Interamericano de Desarrollo [BID], Organización para la Cooperación y el Desarrollo Económicos [OCDE]) también han tenido una incidencia significativa con sus diagnósticos, recomendaciones y propuestas, destacando los problemas de inequidad, baja cobertura, el no cubrimiento de más del 65\% de la población mayor, la sostenibilidad actual del sistema (RPM), el bajo número de fondos privados (4) y su alta concentración en uno o dos. Sus recomendaciones apuntan a elevar la edad de jubilación y una equiparación entre mujeres y hombres, la no competencia entre los dos pilares RPM y RAIS, y que las pensiones estén sujetas al impuesto sobre la renta para los más altos ingresos.

Las otras dos instituciones que han presentado estudios con bastante incidencia en las propuestas de reforma son Fedesarrollo (Leonardo Villar) y el Departamento Nacional de Planeación (DNP) (Mauricio Santamaría). Sus propuestas coinciden, en cuanto a que las cotizaciones de hasta un salario minimo mensual legal vigente (SMMLV) las asuma Colpensiones (RPM) y a partir de este monto, las cotizaciones en calidad de ahorro las administren los fondos privados (RAIS), como única vía para que puedan coexistir los dos pilares y de ser posible, una pensión mínima del $75 \%$ de un salario mínimo (eso implica reformar la Constitución Política). Según Fedesarrollo, se debería contemplar un régimen de transición de entre 10 o 15 años para conservar algunos derechos. En estas propuestas coinciden con Asofondos y ANIF.

En el Gobierno nacional parece haber consenso al respecto, menos en el aumento de la edad para mujeres y hombres y en no aumentar las cotizaciones por lo menos para el empleador. Los derechos adquiridos se van a respetar, la pensión sustitutiva sigue en firme y se revisarán las altas pensiones. En lo demás, el Gobierno aún no se ha pronunciado con claridad, pues le preocupan los planteamientos de los organismos internacionales sobre el $65 \%$ o más de la población no cubierta por el sistema, aunque la solución al respecto se orienta en fortalecer el régimen no contributivo, BEPS y Adulto Mayor, con incremento en parte de la solidaridad de los cotizantes del régimen contributivo.

El Ministro de Hacienda en su intervención en el XII Congreso de la Federación Internacional de Administradores de Fondos de Pensiones (Fiap) y de Asofondos (Cartagena, abril 5 de 2019), expresó que, "En Colombia no habrá reforma pensional, sino reforma a la protección de la vejez", pues la nueva perspectiva amplía el marco del debate más allá del $20 \%$ de la población que hasta ahora ha concentrado las discusiones sobre jubilación en el país, considerando el otro $80 \%$ de la población vieja (en edad de estar jubilada), hasta ahora desprotegido.

Para atender a ese amplio segmento poblacional, el ministro Carrasquilla informó que en marzo de 2019 comenzó a sesionar la Comisión de Reforma de Protección a la Vejez. El Ministerio de Trabajo, de manera conjunta con el Ministerio de Hacienda, trabajan en el proyecto que será presentado a consideración del Congreso en el mes de diciembre de 2019. Esta reforma es de alta sensibilidad para toda la población colombiana (trabajadores formales e informales, cotizantes y no cotizantes, gremios, empresarios, sindicatos, políticos y Gobierno) por sus implicaciones de cobertura, equidad, solidaridad y sostenibilidad del sistema.

En este documento se estudia con mayor profundidad la reforma al sistema pensional formal (RPM y RAIS); es decir, el régimen contributivo, y en menor intensidad el régimen no contributivo (BEPS y Adulto Mayor). Se tratan cinco temas: 1) el contexto histórico y la situación actual del sistema pensional colombiano, 2) las propuestas y tendencias de la reforma del sistema pensional, 3) la propuesta del autor, 4) referencias bibliográficas.

\section{Contexto histórico y situación actual del sistema pensional en Colombia}

Dos hechos significativos incidieron en el origen, evolución y estado actual del sistema general de pensiones en Colombia. El Estado de Bienestar desde la década de los años cuarenta del siglo XX, con una influencia limitada y tardía en el país, y el modelo de economía de mercado con gran incidencia a partir de la Constitución Política de 1991. 


\subsection{El sistema pensional en el Estado de Bienestar (1940-1991)}

La previsión social en Colombia presenta algunos antecedentes a fines del siglo XIX y principios del siglo XX; sin embargo, es hasta la década de 1940, producto del Estado de Bienestar, que se crean dos instituciones oficiales, la Caja Nacional de Previsión Social (Cajanal, 1945) y el Instituto Colombiano de Seguros Sociales (ICSS, 1946), para cubrir a los trabajadores de los sectores público y privado.

Las dos entidades presentaron una cobertura muy limitada y problemas de gestión y financiamiento desde su origen, como consecuencia de los múltiples regímenes especiales, la creación adicional de cajas $\mathrm{y}$ fondos específicos para trabajadores públicos por área de actividad y/o región que fueron agudizando los problemas de sostenibilidad. Además, el ahorro público del ICSS, se convirtió en la caja menor de los gobiernos de turno para financiar programas e instituciones oficiales como el Banco Central Hipotecario, que terminaron desapareciendo. Los gobiernos administraban el ICSS, pero no aportaban al mismo, lo que llevó a los trabajadores a solicitar que se denominara tan solo Instituto de Seguros Sociales, ISS (1977), por cuanto este era sostenido por los aportes de los empleadores y empleados.

\subsection{El modelo de "economía de mercado" y sus implicaciones en el sistema pensional actual}

La adopción del modelo de economía de mercado, imperante desde los años setenta del siglo XX en el mundo occidental, implicó para algunos países de América Latina reformas sociales, económicas y politicas, entre estas la reforma de sus constituciones políticas. Colombia no fue ajena a estos procesos, y en el año 1991 reformó la Constitución Política, la cual contempla tres grandes partes: 1) los derechos y garantías de los ciudadanos; 2) la organización del Estado, y 3) la privatización, con la cual se desmonta el incipiente Estado de Bienestar alcanzado y se acogen los parámetros de la economía de mercado, donde el Estado abandona su papel de productor, proveedor, financiador y distribuidor de bienes y servicios públicos, para asumir una nueva función, la de regulador. A partir de la nueva Constitución Política se crean las diferentes leyes de regulación, como reglas de juego para la participación del sector privado en la producción de los bienes y servicios, cedidos o concesionados al sector privado por el sector público, entre estas la Ley 100 de 1993, de salud y pensiones.

\subsection{El sistema pensional actual (1993-2019)}

En Colombia, mediante la Ley 100 de 1993, se creó el "Sistema de seguridad social integral", y en pensiones está compuesto por dos regímenes excluyentes, RPM y RAIS, que tienen como objetivo cubrir los riesgos de invalidez, vejez y muerte para sus afiliados. Su financiación depende de las cotizaciones sobre el salario, efectuadas por el empleador (12\%) y por los empleados (4\%) durante su vida laboral.

El número de afiliados al Sistema General de Pensiones a diciembre de 2018 era de 22.195.779, de las cuales el 30,2\% (6.704.364) corresponden al RPM y el 69,8\% (15.491.415) al RAIS. El crecimiento fue del 4,3\% de 2017 en relación con el año 2018. La distribución se muestra en la tabla 1.

Tabla 1. Afiliados al Régimen General de Pensiones, total a dic. 2018

\begin{tabular}{lrrrrr}
\multicolumn{2}{c}{$\begin{array}{c}\text { Régimen de ahorro individual (RAIS): } \\
\text { Fondos privados }\end{array}$} & & \multicolumn{2}{c}{$\begin{array}{c}\text { Régimen de Prima Media (RPM): } \\
\text { Instituciones oficiales }\end{array}$} \\
\hline PORVENIR: & 8.865 .068 & $40,0 \%$ & COLPENSIONES: & 6.702 .549 & $30,2 \%$ \\
\hline PROTECCIÓN: & 4.602 .047 & $21,0 \%$ & FONPRECON: & 946 & $0,004 \%$ \\
\hline COLFONDOS: & 1.909 .752 & $8.6 \%$ & F. ANTIOQUIA: & 413 & $0,002 \%$ \\
\hline OLD MUTUAL: & 114.237 & $0,5 \%$ & CAXDAC: & 456 & $0,002 \%$ \\
\hline $\begin{array}{l}\text { OLD MUTUAL } \\
\text { Alternativo: }\end{array}$ & 311 & $0,001 \%$ & & & \\
\hline PORVENIR: & 15.491 .415 & $69,8 \%$ & TOTAL AFILIADO RPM: & 6.704 .364 & $30,2 \%$ \\
\hline
\end{tabular}

Total Afiliados al Sistema General de Pensiones: 22.195.779

Fuente: diseño del autor con base en "Informe de Gestión”, Colpensiones, 2018. 
Tabla 2. Distribución de afiliados por rango salarial, RPM

\begin{tabular}{lrr}
\hline Rango SMMLV & Cantidad & Porcentaje \\
\hline Menor a 2 SMMLV & 5.977 .873 & $89,19 \%$ \\
\hline >2 y 4 SMMLV & 381.488 & $5,69 \%$ \\
\hline >4 y 7 SMMLV & 194.982 & $2,91 \%$ \\
\hline >7 y 10 SMMLV & 77.972 & $1,16 \%$ \\
\hline >10 y 13 SMMLV & 30.001 & $0,45 \%$ \\
\hline >13 y 16 SMMLV & 16.681 & $0,22 \%$ \\
\hline >16 SMMLV & 25.552 & $0,38 \%$ \\
\hline TOTAL & 6.702 .549 & $100,00 \%$
\end{tabular}

Fuente: Informe de gestión 2018, Colpensiones, Gerencia de Planeación Institucional

Colpensiones cuenta con 6,7 millones de afiliados, 700.000 más que los que recibió del liquidado ISS en 2012 , lo cual equivale al $30,2 \%$ de los 22 millones de colombianos afiliados al sistema pensional (tabla 2). Los requisitos exigidos para obtener una pensión en RPM a partir del año 2014 se modifican, así: edad mínima de 62 años para los hombres y 57 para las mujeres, y en ambos casos haber cotizado 1.300 semanas (aproximadamente cerca de 25 años).
El Informe de gestión de Colpensiones (2018) relaciona un total de 1.486 .827 pensionados a diciembre de 2018 en Colombia, con un crecimiento del $4,5 \%$ con respecto al mismo periodo del año 2017. El 89,3\% (1.334.956) de los pensionados corresponden al régimen de prima media y el $10,7 \%(151.871)$ al régimen de ahorro individual. La distribución se presenta en la tabla 3.

Tabla 3. Pensionados Régimen Sistema General de Pensiones a dic. 2018

\begin{tabular}{lrlr}
\hline $\begin{array}{l}\text { Régimen de ahorro individual (RAIS): } \\
\text { Fondos privados }\end{array}$ & \multicolumn{2}{c}{$\begin{array}{c}\text { Régimen de Prima Media (RPM): } \\
\text { Instituciones oficiales }\end{array}$} \\
\hline PORVENIR: & 69.691 & COLPENSIONES: & 1.328 .986 \\
\hline PROTECCIÓN: & 54.076 & FONPRECON: & 2.389 \\
\hline COLFONDOS: & 24.407 & F. ANTIOQUIA: & 2.852 \\
\hline OLD MUTUAL: & 3.532 & CAXDAC: & 729 \\
\hline OLD MUTUAL ALTERNATIVO: & 165 & & 1.334 .956 \\
\hline Total Pensionados AFP: & 151.871 & Total Pensionados RPM: & \\
\hline Total Pensionados del Sistema General de Pensiones: 1.486 .827 &
\end{tabular}

Fuente: informe de gestión 2018, Colpensiones, Gerencia de Planeación Institucional.

Tabla 4. Distribución de pensiones por nivel salarial RPM

\begin{tabular}{lrr}
\hline \multicolumn{1}{c}{ Rango salarial } & Número de pensionados & Porcentaje \\
\hline Menos de 2 SMMLV & 1.015 .759 & $76,43 \%$ \\
\hline Entre 2 y 4 SMMLV & 200.807 & $15.11 \%$ \\
\hline Entre 4 y 7 SMMLV & 77.608 & $5,46 \%$ \\
\hline Mayor a 7 SMMLV & 39.812 & $3,00 \%$ \\
\hline Total & 1.328 .986 & $100,00 \%$
\end{tabular}

Fuente: Informe de gestión 2018, Colpensiones, Gerencia de Planeación Institucional

En relación con las pensiones reconocidas y pagadas por Colpensiones, 1.015.758 pensionados (75,43\% del total) tienen asegurada una pensión inferior a los 2 SMMLV; un 15,11\% (200.807 pensionados) entre 2 y 4 salarios; el 5,46\% (77.608) entre 4 y 7 SMM, y un 3\% (39.812) de pensionados recibe una pensión superior a los 7 SMMLV (tabla 4). Como ingreso, 303.185 pensionados reciben en promedio \$916.991; algo más del 10\% (125.482) tienen en promedio una pensión de $\$ 1.568 .526$, y en más del 7\% (93.013) de personas, las pensiones superan los dos millones de pesos en promedio.

Según el Informe de gestión de Colpensiones (2018), los recursos que recibe, producto de los aportes de sus afiliados que cotizan para su futura pensión, se destinan de manera inmediata al pago 
de las mesadas de quienes hoy están pensionados por el RPM. Por lo tanto, este ahorro no genera rentabilidad, por lo que crece en la medida en que se hagan los respectivos aportes.

\subsection{Régimen no contributivo: Beneficios Económicos Periódicos (BEPS) y Adulto Mayor}

Los BEPS es un programa de ahorro voluntario diseñado para proteger a las personas cuyos recursos o ingresos no les alcanza para cotizar a una pensión. Las personas que ahorran en BEPS, construyen un capital que les permitirá disfrutar en su vejez de un ingreso económico de por vida. $\mathrm{El}$ programa es flexible y permite a los ciudadanos ahorrar de acuerdo con sus capacidades económicas de manera diaria, semanal o mensual.

Se pueden afiliar al programa los ciudadanos colombianos, mayores de 18 años, con ingresos inferiores a un SMMLV, a través de Colpensiones o de un Fondo Privado de pensiones. Si el afiliado utiliza los recursos ahorrados en BEPS y sus rendimientos, para completar el número de semanas mínimas requeridas y acceder a su pensión, en este evento tendrá derecho al 20\% del incentivo periódico; es decir, al 20\% de los aportes realizados por el beneficiario.

$\mathrm{El}$ incentivo es un subsidio que consiste en un aporte económico otorgado por el Estado y se calcula anualmente de manera individual para cada beneficiario sobre los aportes que haya realizado; de esta manera, se constituye en un apoyo al esfuerzo de ahorro. La normatividad vigente estableció un monto mínimo de ahorro de $\$ 5.000$ y máximo al año de \$990.000 para el año 2017 . El total de ciudadanos vinculados a los BEPS a diciembre 31 de 2017 fue de 937.458. La población rural colombiana viable para vincular a BEPS se estima en 3.075.274 ciudadanos, de los cuales, el $76 \%$ se concentra en 10 departamentos, con mayor participación Antioquia (13.2\%), Cundinamarca (8.9\%) y Santander (8.2\%).

\section{Las propuestas y las tendencias de la reforma del sistema pensional en Colombia}

Las propuestas de reforma se presentan desde diferentes intereses y perspectivas. Las más publicitadas e influyentes provienen del sistema financiero nacional e internacional propietario de los fondos privados organizados en Asofondos, y de la Asociación de Instituciones financieras (ANIF), que también representa y defiende los intereses del sector financiero. Los organismos internacionales están interesados en afianzar el modelo de economía de mercado, la privatización, la eficiencia y la gestión pública por resultados (eficacia del gasto y reducción de la pobreza). Los centros de investigación y consultoría como Fedesarrollo y Fescol, con sus propuestas apuntan a favorecer también esos intereses. La Sindical Obrera es proclive a conservar en parte el régimen público (RPM), a favorecer sus intereses y a la inclusión de la población marginada del sistema de seguridad; mientras el Gobierno, interviene con instituciones como el Departamento Nacional de Planeación (DNP). El Ministerio de Hacienda, en menor escala Colpensiones, y el Ministerio de Trabajo, coordinador de la reforma, parecen más receptivo a las propuestas de Asofondos y de Fedesarrollo.

\subsection{La propuesta de los organismos internacionales: FMI, BID, OCDE}

Las calificadoras internacionales de riesgo y entidades multilaterales como el Fondo Monetario Internacional (FMI), Banco Interamericano de Desarrollo (BID), el Banco Mundial y la Organización para la Cooperación y el Desarrollo Económico (OCDE), desde el primer semestre del año 2018 han mANIFestado su preocupación por la demora en la toma de decisiones sobre la reforma pensional.

\subsubsection{Fondo Monetario Internacional (FMI)}

La misión del Fondo Monetario Internacional en la visita de evaluación de abril de 2018, advirtió que Colombia necesita "una reforma integral del sistema pensional para mejorar la cobertura y la progresividad", y desactivar la "bomba social a punto de estallar” (El Nuevo Siglo, marzo 05 de 2018). Para el FMI, el sistema de pensiones tiene una baja cobertura y es altamente regresivo: "Por eso reducir las tasas de reemplazo y aumentar las edades de jubilación de hombres y mujeres serían pasos importantes para fortalecer el sistema pensional". Es fundamental elevar la edad de jubilación de los colombianos, como uno de los puntos clave de una reforma, no de manera inmediata sino de forma gradual, para no impactar la economía de los colombianos. Se requiere un aumento en la edad de retiro y una equiparación, es decir, acercamiento entre hombres y mujeres, también de manera gradual, por el interés de la gente en conocer a qué edad se va a pensionar. 
Deben considerarse reformas estructurales más profundas del sistema contributivo con el fin de ampliar el acceso a las pensiones, ya sea que estén gestionadas por Colpensiones o por el sistema de ahorro privado. Las medidas que se tomen deberán propender por garantizar la "sostenibilidad financiera del sistema", pues el pago de pensiones sumará unos 41,1 billones de pesos en el PGN 2018, lo que equivale al $17,5 \%$ del presupuesto general del año 2018. Además, los sistemas pensionales actuales, prima media y ahorro individual, no deberían competir entre ellos. La misión recomienda que se implante una pensión no contributiva que cubra a los pobres, para combatir la pobreza.

En la visita de evaluación, un año después (marzo de 2019), la segunda observación del FMI tiene que ver con la necesidad urgente de realizar una reforma pensional de fondo, e insiste en elevar en forma prudente las edades de jubilación, tal como está aconteciendo en todos los países avanzados, para ajustarse al hecho innegable de que la vida promedio de los ciudadanos se ha elevado, y de que la mayoría de los jubilados cercanos a los 60 años, bien pueden todavía laborar no menos de cinco años más e incrementar de paso su mesada pensional (El Espectador, marzo 22 de 2019).

\subsubsection{Organización para la Cooperación y el Desarrollo Económico (OCDE)}

Un capítulo del informe de la OCDE (2015) sobre Colombia, se encuentra dedicado al tema de las pensiones. Para la OCDE se requiere "aumentar la edad de jubilación de manera gradual” porque, aunque hay pilares privados y públicos, "el problema es que el sistema público no cubre a los más necesitados y por eso no disminuyen las brechas". El sistema pensional tiene una cobertura baja y "beneficia a los que tienen más, y los pasos dados en los últimos años como Colombia Mayor son importantes; por eso se debe expandir Colombia Mayor y los Beneficios Económicos Periódicos (BEPS)" (OCDE, 2015).

Se destaca en el informe que por la Constitución Política (1991), las pensiones mínimas no pueden ser inferiores al salario mínimo, y eso implica que solo los trabajadores formales cuyo salario es igual o superior al mínimo pueden aspirar a recibir una pensión. Sin embargo, resalta que exista el esquema de Beneficios Económicos Periódicos (BEPS) para los trabajadores informales, quienes no han podido acceder a una pensión de salario mínimo, ya sea por la intermitencia de su trabajo, o porque su salario ha sido inferior al mínimo. Se destaca el otro mecanismo de apoyo a la vejez denominado ahora "Colombia Mayor", orientado a los colombianos pobres quienes desarrollaron su vida laboral en el sector informal y han cumplido la edad de pensión.

En el diagnóstico sobre los retos que enfrenta el sistema pensional colombiano, critica su bajo nivel de cobertura, pues solo el 37\% de quienes llegan a edad de pensionarse reciben una pensión, lo cual ubica a Colombia inclusive por debajo de la media regional. Esa baja cobertura refleja la informalidad laboral y la restricción constitucional descrita.

En las recomendaciones resalta los esquemas BEPS, Adulto Mayor, la pensión conyugal y familiar e invita a repensar la conveniencia de conservar la restricción constitucional ya referida. En cuanto al RPM de Colpensiones, critica lo generoso e inequitativo del mismo, y que las pensiones no están sujetas al impuesto sobre la renta favoreciendo a los pensionados que tuvieron los más altos ingresos. Así mismo, señala el hecho del bajo número de años de cotización exigidos para pensionarse y las edades de pensión, los cuales deben ser revisados al alza; recomienda que a futuro y de manera gradual se iguale la edad de pensión para hombres y mujeres, dada la mayor expectativa de vida de las mujeres.

Sobre el sistema contributivo de los fondos privados de pensiones, su análisis, críticas y recomendaciones se refieren a la concentración de los fondos privados de pensiones, solo existan cuatro, y el $80 \%$ de los ahorros se concentra en dos. Sin embargo, destaca el rol de los fondos privados de pensiones en el ahorro financiero y su adecuado mecanismo de operación, los diversos fondos de riesgo a los que pueden acceder los ahorradores, y el sistema del seguro previsional que contratan los fondos de pensiones con las aseguradoras para cubrir los eventuales riesgos de incapacidad y sobrevivencia de sus ahorradores.

El Informe de la OCDE (2015) finaliza destacando la importancia de establecer un régimen más ambicioso de pensiones, y como medida estructural propone gradualmente marchitar el RPM, sugiriendo que los nuevos trabajadores estén obligados a vincularse al sistema contributivo de los fondos privados. 


\subsubsection{Banco Interamericano de Desarrollo (BID)}

Colombia necesita una reforma del sistema pensional, y su objetivo se debe centrar en solucionar los problemas de la falta de cobertura, alta inequidad del sistema, potenciales riesgos de sostenibilidad fiscal dada la transición demográfica, competencia desigual entre el régimen de prima media (RPM) y el régimen de ahorro individual con solidaridad (RAIS), y la mejora del funcionamiento de las instituciones relacionadas con el sistema pensional (Berstein et al., 2015, p. 1).

Los principales problemas del sistema pensional colombiano son, a juicio del BID (Berstein et al., 2015):

- La deficiencia institucional: falta una institución que dé rectoría al sistema pensional en su conjunto y coherencia a sus distintas partes.

- Alto costo: en 2013 el Gobierno colombiano cubrió un déficit del RPM de 3,8\% del PIB para subvencionar al 37\% de adultos de 60 años y más con pensiones contributivas. Conforme la cobertura baje en el tiempo, el déficit se situará en $2,1 \%$ del PIB en 2050 y 1,7\% en 2075.

- Alta inequidad: el $80 \%$ de los subsidios implícitos del Estado al sistema de pensiones los recibe el $20 \%$ de la población de mayores ingresos. Estos subsidios para los afiliados de mayor ingreso pueden llegar en valor presente a los 1.000 millones de pesos (US\$ 420.000) por afiliado.

- El deficiente funcionamiento del régimen privado de pensiones (RAIS) en su parte de desacumulación.

- Un alto riesgo jurídico: no hay una clara interpretación jurídica de las leyes, decretos y normas que rigen el sistema pensional, lo que provoca que los agentes e instituciones del sistema pensional operen bajo una alta incertidumbre.

La problemática del sistema pensional colombiano se asienta en un mercado de trabajo de alta informalidad que hace que cualquier diseño no pueda proporcionar una cobertura aceptable. Dada la estructura actual del sistema colombiano, una reforma consistente con una visión a largo plazo requiere: (i) considerar el sistema pensional en su conjunto; (ii) incrementar la cobertura; (iii) mantener un equilibrio entre contribuciones y beneficios; (iv) reducir la inequidad; (v) proporcionar subsidios apropiados, progresivos y sostenibles.

\section{Recomendaciones y propuestas}

\section{A. Incrementar la cobertura del sistema:}

a. Expandir el apoyo a la vejez provisto por Colombia Mayor para establecer un pilar no contributivo universal anti-pobreza, con una fuerte institucionalidad que asegure su sostenibilidad.

b. Ampliar y potenciar la opción de acceder a las rentas vitalicias ofrecidas por el BEP para todos los que hoy aportan al sistema contributivo (RAIS o RPM), pero que no calificarán para una pensión.

c. Implementar acciones paralelas a la reforma pensional con el foco en la creación de trabajo formal.

B. Afrontar reformas importantes de funcionamiento del sistema privado para que se facilite la etapa de desacumulación y de viabilidad a la industria de pensiones privada.

C. Es crucial implementar reformas paramétricas al sistema para mitigar la inequidad existente, asegurar la sostenibilidad en el mediano plazo, reducir los desajustes de beneficios entre el RAIS y el RPM y permitir una expansión cobertura contributiva.

D. Mejoras institucionales del sistema pensional y reducción del riesgo jurídico.

E. Afrontar el rediseño del sistema pensional eliminando la competencia entre regímenes contributivos. Esto implica reformar el régimen de prima media hasta marchitarlo completamente o, alternativamente, convertirlo en un pilar de beneficio definido básico.

\subsection{Las propuestas del sistema financiero colombiano: Asofondos y ANIF}

El sector financiero, propietario de los fondos privados de pensiones, organizados en la Asociación de Fondos Privados (Asofondos) y la Asociación Nacional de Instituciones Financieras (ANIF), desde su creación en 1974, han jugado un papel destacado en la defensa de la política económica e intereses del sector, a través de estudios y reflexiones con amplia influencia en la opinión pública y en los dirigentes. 


\subsubsection{Asociación Colombiana de Administradoras de Fondos de Pensiones y Cesantias (Asofondos)}

- Las ideas generales de la propuesta de Asofondos son las siguientes (Montenegro, 2018):

- En las condiciones actuales y futuras de Colombia, el único sistema pensional contributivo viable debe estar basado en el régimen de ahorro y capitalización (RAIS).

- Un sistema de reparto puro no es viable por la transición demográfica, la informalidad laboral y la digitalización de la sociedad (mayor nivel de automatización de la sociedad, robots).

- La llamada solidaridad intergeneracional, que dio lugar a los sistemas de reparto, está rota: cuando sean adultos mayores, no habrá quién les pague las pensiones a los jóvenes de hoy.

- La solidaridad debe ir focalizada a quienes realmente sí lo necesitan, y debe estar financiada con presupuesto público.

- El sistema contributivo, que deberá ser de ahorro y capitalización, podrá ser administrado por entes públicos y privados, que compitan bajo las mismas reglas.

- La reforma que se implemente deberá respetar los derechos adquiridos de los retirados y las expectativas legítimas de quienes estén próximos a jubilarse.

Para Asofondos, los problemas de sistema pensional se caracterizan por: 1) en su componente contributivo, es muy inequitativo; 2) en su componente de pensiones, tiene un déficit fiscal muy alto; 3) una bajísima cobertura, tiene completamente desprotegidos a 2,8 millones de ancianos y existen 5,5 millones de adultos mayores de 60 años, de los cuales:

- 2.794.133 no cuentan ni con pensión ni con subsidio (0 pesos).

- 1.473.574 tienen acceso a Colombia mayor (\$75.000 por mes, US\$ 25/mes).

- 1.274.770 tienen pensión (vejez, invalidez o sobrevivencia).

En cuanto a la insostenibilidad, se evidencia que el sistema de reparto no es compatible con un aumento de formalidad, entre mayor formalidad, mayor déficit pensional (una persona que cotiza 500 semanas por un salario mínimo recibe en RAIS $=36,7$ millones, en RPM = 12,9 millones a una tasa promedio de $5 \%$ real).

Las bases para una reforma, según Asofondos, son las siguientes:

- Por las razones expuestas, el sistema pensional debe ser de ahorro y capitalización.

- El debate debe ser entre los sistemas viables y los no viables, no entre si lo administra el Gobierno o el sector privado. Lo pueden administrar ambos, bajo las mismas reglas.

- Para quienes no tengan capital suficiente, se plantea el acceso a una pensión de salario mínimo con 1.150 semanas cotizadas y que cumplan con la edad legal de retiro.

- Se deben respetar los derechos adquiridos y las expectativas legítimas de quienes estén próximos a jubilarse: a partir de cierta fecha, los nuevos cotizantes entrarán solo a un régimen de capitalización, con administradores privadas y públicas, y para quienes les falten más de 10 años para el retiro se definirá un régimen de transición.

En concreto, Asofondos propone que Colpensiones compita en el mercado como una AFP más, es decir, poner fin al régimen de prima media. También propone fortalecer los BEPS para los más pobres y los trabajadores informales de las zonas rurales, así como la ampliación de la cobertura del programa "Colombia Mayor".

\subsubsection{Asociación Nacional de Instituciones Financieras (ANIF)}

La propuesta de reforma estructural pensional de ANIF consiste en dos pilares: 1) cotizaciones de hasta un SMMLV para obtener una pensión mínima de $75 \%$ del mismo y, 2) ahorro individual adicional para ingresos superiores a un SMMLV. La persona puede ahorrar adicionalmente al salario mínimo para mejorar su pensión en cualquiera de los dos fondos (privado o público). Se requiere también aumentar la cotización, aumentar la edad de pensión a partir del año 2028, un año adicional por año para hombres y mujeres, hasta llegar a una edad 62 años para las mujeres y 67 años para los hombres en el año 2034. Con la propuesta se reduce el subsidio del gobierno de 68,3\% a 53,6\% hacia el año 2050 y se marchita el RPM. 
Adicionalmente, ANIF presentó una propuesta de reforma a la instalada "Comisión de Protección a la Vejez" creada por el Gobierno en marzo de 2019 (Portafolio, junio 10 de 2019), en la cual sugiere lo siguiente:

- Aumentar escalonadamente la edad de pensión, reducir la tasa de reemplazo, permitir que las personas se jubilen con el $75 \%$ de un salario mínimo y suspender las nuevas afiliaciones a Colpensiones para que se vaya marchitando el esquema público, son las propuestas para una reforma estructural al sistema pensional. También sería deseable que la pensión mínima se pudiera reducir al 75 por ciento del SMMLV, como ocurre en Chile. Lo mismo aplicaría para la pensión sustitutiva a los sobrevivientes, con excepción de aquellos casos en los que los beneficiarios sean menores de 25 años. Se plantea una transición amplia (entre 10 y 15 años), con el fin de respetar los derechos adquiridos.

- En cuanto a la edad, ANIF propone aumentarla en cinco años como requisito para pensionarse, con lo cual pasaría de 57 a 62 años en el caso de las mujeres, y de 62 a 67 años para los hombres, aunque dicho incremento sería gradual. ANIF calcula que la reducción en el tiempo de disfrute de la pensión (pasaría de 30 a 25 años, en promedio), generaría un alivio fiscal de 7,4 puntos del PIB entre 2020 y 2050, que en los siguientes años se elevaría a 16,7 puntos.

- El segundo ajuste propuesto tiene que ver con la tasa de reemplazo (monto máximo de pensión), es decir, lo que una persona recibe de pensión como proporción del salario con el que aportó. Actualmente se parte de una base del $65 \%$ (más $1,5 \%$ por cada 50 semanas adicionales) hasta un monto máximo de $80 \%$ del Ingreso base de liquidación, IBL. Según ANIF, la base debe reducirse en 20 puntos porcentuales, a $45 \%$ "que es la cifra que actualmente pueden garantizar portafolios invertidos a tasas del orden del 6 por ciento real por año durante 25 años de cotizaciones" (Clavijo, 2017).

- Advierte que es fundamental ir trabajando en una reforma estructural laboral para elevar la formalidad, especialmente en los jóvenes, quienes enfrentan desafíos crecientes por la situación actual de los trabajos. En materia educativa, ANIF propone que los estudiantes puedan elegir entre el bachillerato clásico y una opción vocacional: "Allí, será fundamental evaluar la calidad y pertinencia de dichos programas vocacionales. Sin buena calidad educativa, tampoco será posible incrementar la productividad y el PIB potencial de Colombia” (Clavijo, 2017).

\subsection{Centros de investigación y consultoria: Fedesarrollo y Fescol}

La Fundación para la Educación Superior y el Desarrollo (Fedesarrollo) es una entidad privada sin ánimo de lucro, establecida en 1970. Se dedica a la investigación en temas de política económica y social. Su propósito es contribuir al diseño, seguimiento y mejoramiento de las políticas públicas.

\subsubsection{Fedesarrollo (Forero y Villar)}

- Como diagnóstico señalan los problemas del actual esquema, vistos desde la perspectiva de los cuatro principios de todo sistema pensional: cobertura, equidad, solidaridad y sostenibilidad. Más adelante, los autores afirman que "El sistema pensional colombiano que se originó en la reforma de la Ley 100 de 1993, no ha logrado solventar de forma satisfactoria las funciones de protección social que debe cumplir. Presenta problemas de baja cobertura, ineficacia de los mecanismos de solidaridad, inequidad en los subsidios otorgados, y un alto costo fiscal" (Villar y Forero, 2018, p. 11)

- Cobertura. El sistema pensional actual cuenta con una cobertura de afiliados que oscila entre el 32 y 34\% de la población económicamente activa, el RPM cubre el 9,5\% y el RAIS, $25,1 \%$. Los bajos niveles de cobertura tanto en porcentajes de cotizantes como del adulto mayor obedecen en buena parte a la incidencia de la informalidad laboral en Colombia.

- Equidad. Según los autores, el sistema pensional es inequitativo y regresivo. El RPM es un sistema subsidiado, pero este lo reciben en alto porcentaje los quintiles 4 y 5 .

- Solidaridad. Según Forero y Villar (2018, p.20):

"Los mecanismos de solidaridad del sistema pensional en Colombia se materializan en el Fondo de Garantía de Pensión Mínima (FGPM) del RAIS, y el Fondo de Solidaridad Pensional 
(FSP). Este último constituye la fuente de financiación del Programa Colombia Mayor desde 2003, financiado por aportes entre uno y dos puntos porcentuales del ingreso base de cotización de los trabajadores de ingresos mayores a cuatro salarios mínimos de ambos regímenes.

Como el RPM concede la pensión sobre el promedio de los diez últimos años de trabajo, sus afiliados obtienen una mejor pensión, de ahí que los afiliados de altos ingresos se trasladen del RAIS al RPM para obtener los subsidios del gobierno.

- Sostenibilidad. Al respecto Forero y Villar (2018, p. 20), agregan que:

"En la actualidad, las pensiones otorgadas por los regímenes públicos (RPM + Especiales) no alcanzan a ser financiadas con las cotizaciones anuales de los trabajadores, por lo que el Estado tiene que dirigir una parte considerable del Presupuesto General para cubrir el faltante".

- Envejecimiento. El peso de los adultos mayores en el total de la población se triplicará en los próximos 30 años, pasando de $7 \%$ en 2015 a $21 \%$ en 2050.

En síntesis, la base de la propuesta de reforma descansa en la integración entre el régimen de prima media (pilar 1) y el régimen de capitalización individual (pilar 2). El principio esencial de la reforma pensional es acabar con la lógica de competencia que opera actualmente entre los dos regímenes contributivos que se disputan la cobertura de la misma población: los trabajadores formales. El propósito de la reforma debe ser crear los mecanismos para que dichos regímenes actúen con un criterio de complementariedad entre ellos. Esto significa que se acabaría el concepto de "afiliado" a uno u otro esquema, y todos los trabajadores formales estarían afiliados a ambos regimenes al mismo tiempo. Dado que en un esquema multipilar los diferentes componentes cumplen funciones diferenciadas y excluyentes, la propuesta no considera eliminar ninguno de los actuales esquemas. De esta forma, el nuevo sistema funcionaría segmentando los aportes de cada individuo entre los dos regimenes contributivos, y cada pilar cumpliría una función diferente y suplementaria (Forero y Villar, 2018, p. 29).

El Pilar 1, controlado por el Estado bajo el esquema de Prima Media a través de Colpensiones, recibiría los aportes de todos los trabajadores correspondientes a 1 SMMLV, y se encargaría de otorgar el primer tramo de la prestación pensional, el cual garantizaría una pensión equivalente a 1 SMMLV mediante pagos de esa magnitud para quienes cumplen los requisitos de pensión pero no tienen ahorros en el régimen de capitalización individual, y pagos complementarios a los de dicho régimen para quienes tienen dichos ahorros (Forero y Villar, 2018, p. 29). Lo anterior, según los autores, elimina de plano la concentración de los subsidios del Estado en la población de altos ingresos.

El Pilar 2, manejado por las Administradoras de Fondos de Pensiones Privadas (AFP) bajo la lógica de cuentas de ahorro individual, se encargaría de mejorar las pensiones de aquellos individuos con mayor capacidad de ahorro en su etapa laboral. Así, recibirían toda cotización que supere el aporte equivalente a 1 SMMLV, lo acumularían en la cuenta individual del trabajador, y se encargarían de otorgar el segundo componente de la pensión, esto es, el excedente sobre 1 SMMLV mediante la modalidad de rentas vitalicias (Forero Villar, 2018, p. 30).

En cuanto a las características de la transición, a los pensionados actuales y los trabajadores a los que les falten menos de diez años para la edad de pensión se los mantenga en el régimen que tienen, sin cambio alguno. Para aquellos trabajadores nuevos que ingresen al mercado laboral y para los que les falten más de 20 años para la pensión sería obligatorio acogerse al sistema de cotizaciones por pilares complementarios. Finalmente, para los trabajadores activos a los que les faltan más de 10 años, pero menos de 20 años para la edad de pensión, debería dárseles un plazo para escoger de manera definitiva entre mantenerse en el esquema actual de RAIS, mantenerse en el sistema de Colpensiones o entrar al esquema multipilar planteado en este documento (Forero y Villar, 2018, p. 42).

Adicional a lo anterior, Fedesarrollo propone:

- Un ajuste inicial a las edades de pensión, que se incremente para ambos sexos y reduzca la brecha entre ellos, por ejemplo, acercándolas gradualmente hacia los 65 años para todos 
aquellos cotizantes a los que hoy les faltan más de 20 años para pensionarse.

- Crear un mecanismo automático de ajuste periódico (por ejemplo, cada quinquenio) de la edad de pensión en función de la expectativa de vida o de otros parámetros de la evolución demográfica del país.

- Unificar los requisitos mínimos para pensión, que hoy en día son diferenciados, en materia de número de semanas cotizadas (RPM-1.300, RAIS-1.150).

- Finalmente, la tasa de cotización debería unificarse en $18 \%$, tal como lo propuso recientemente la comisión del gasto y la inversión pública en su informe final al Gobierno nacional (Comisión de Gasto Público, 2018). Ello reduciría de manera importante el déficit actuarial del RPM.

\section{Propuestas de reforma al programa de beneficios económicos periódicos (BEPS)}

El concepto de BEPS fue incorporado en la Constitución Política en 2005, pero solamente hasta una década después comenzó su implementación. Si el programa mantiene su estructura actual, enfocado estrictamente en ahorro voluntario y con cobertura focalizada a través de diversos instrumentos, es improbable que genere un impacto estructural sobre el sistema de protección económica a la vejez. En primer lugar, los BEPS están focalizados sobre la población con salarios menores a 1 SMMLV. Segundo, el hecho de que el usuario tenga que ganar menos de 1 SMMLV excluye individuos que reciben más de este monto, pero de diferentes fuentes o los que no cuentan con contrato de trabajo. Tercero, se está dejando por fuera de los beneficios del programa todos aquellos trabajadores que, con un salario mayor a 1 SMMLV, aportan al sistema, pero no alcanzan los requisitos para una pensión contributiva, por lo que reciben una devolución de saldos o indemnización sustitutiva y no la pueden convertir en una renta vitalicia BEPS, dado que se salen del criterio de focalización (Forero y Villar, 2018, p. 50).

Un buen sistema de protección económica para la vejez debe cumplir dos objetivos: el primero es el de aseguramiento, en el que actúa como sustitución del ingreso de los trabajadores en su etapa pasiva, por lo que mejores salarios implicarían mejores pensiones; el segundo es el asistencial, en el que se alivia la pobreza de la población mayor más vulnerable, y donde por definición los que reciben el subsidio son los individuos que menos impuestos han pagado a la sociedad. Estos son los pilares Contributivo (Uno y Dos) y No Contributivo (pilar Cero) del sistema, respectivamente; y que, además, existen en Colombia (Forero y Villar, 2018, p. 59).

\subsubsection{Friedrich Ebert Stiftung en Colombia Fescol)}

Arrieta (2011) relaciona un documento de la Escuela Nacional Sindical (2005), y con base en el mismo argumenta que, "el desbalance financiero del sistema pensional se dio no por aquellos regímenes conquistados por las labores sindicales sino por los que se establecieron por abusos de poder". Es decir, por "los beneficios de los regímenes especiales los cuales algunos como el de Ecopetrol fueron establecidos por conquistas sindicales, mientras que otros como el de la Presidencia de la República, el del Congreso de la República, las Altas Cortes, el Banco de la República y las Fuerzas Armadas fueron logradas por el aprovechamiento del poder".

En conclusión, la consecuencia principal de las reformas vigentes al sistema pensional colombiano es la racionalización de los recursos públicos. En este orden, se busca una sostenibilidad financiera, la cual no fue lograda por medio de la Ley 100 de 1993. El Acto Legislativo No. 1 de 2005 dejó vigentes los regímenes especiales de la Presidencia de la República y las Fuerzas Armadas, que constituyen el mayor peso fiscal al sistema pensional colombiano. Se recomienda crear un sistema pensional en donde los parámetros sean generales para todos y cada uno de los trabajadores colombianos, en donde realmente sea efectivo el principio de equidad.

\subsection{La propuesta de la Unión Sindical Obrera (CUT y CTC)}

Las propuestas de estas dos centrales sindicales apuntan al derecho a la protección, a la garantía de ingresos para toda la población adulta mayor bajo la noción de dignidad, en la que supuestamente descansa el Estado colombiano. Proponen rescatar el sistema pensional como un sistema público de protección social, cuyo objetivo es garantizar derechos humanos fundamentales y no la posibilidad de hacer negocios, como ocurre hoy 
con el sistema de ahorro individual. Debe existir coherencia entre el sistema contributivo y el no contributivo, y entre las instituciones encargadas del sistema de protección a la vejez. Más de dos tercios de las personas que llegan a edad de pensión reciben devolución de saldos porque es insuficiente su capital o las semanas cotizadas. En el caso de las mujeres las devoluciones doblan a las de los hombres. Las devoluciones en el RAIS constituyen el $75 \%$, y en Colpensiones el 50\%.

En Colombia el modelo pensional se diseñó para que solo se pensionen personas que trabajen en el sector formal de la economía, tengan estabilidad laboral, altos niveles educativos y trabajen preferiblemente en zonas urbanas. De ahí que apenas el 30\% de la población mayor de 60/62 años tiene acceso a una pensión, y del total de afiliados al Sistema General de Pensiones (SGP) solo el 38\% son cotizantes (7,7 millones). En el sector rural la afiliación a pensiones es menor al $10 \%$. Es larga la lista de trabajadores excluidos del SGP: los del sector informal, en su mayoría los cuentapropia, las personas con discapacidad, las "amas de casa”, las y los trabajadores temporales sin estabilidad laboral, la mayoría de los trabajadores del campo, y la mayoría de las personas pobres (88\%), que en el nivel más bajo cotizan en promedio 5 semanas por año.

En cuanto a los dos regímenes pensionales que coexisten, el desbalance en favor del régimen privado es desproporcionado. El Régimen de Ahorro Individual (RAIS) en manos de las Administradoras de Fondos de Pensiones (AFP), en 2017 tenía \$227 billones de ahorro pensional, proveniente de 14,8 millones de afiliados, y cargaba con solo 137.926 pensionados. El $40 \%$ de estos recursos se utilizan como deuda pública del Estado. Mientras que el Régimen de Prima Media (Colpensiones), con \$5,8 billones de aportes y 6,5 millones de afiliados, carga con 1,2 millones de pensionados. Por lo que el Estado debe destinarle $\$ 13$ billones para que pueda pagar el déficit de Colpensiones y $\$ 40$ billones para todas las pensiones a cargo del Estado.

La reforma del régimen de pensiones que necesita el país debe partir de las orientaciones de Naciones Unidas y la OIT, en el sentido de que los países adopten un piso de protección social adaptado a las necesidades y contextos locales, que pueda implementarse de forma progresiva. Entendido este piso como el acceso a una canasta básica de bienes y servicios que asegure que la gente no sufra hambre ni enfermedades evitables, no se quede sin educación, sin vivienda ni agua potable.

Según la Sindical Obrera, su propuesta es similar a otras que se han planteado en otros países como estrategia eficaz para disminuir los indices de pobreza en el planeta y hacer frente a los períodos de crisis económicas. Naciones Unidas y OIT propusieron la adopción de "pisos de protección social”, propuesta que incluye como beneficiarias del sistema pensional a las mujeres responsables de la economía del cuidado en los hogares, como una forma de reparar su exclusión y reconocer la importancia de su trabajo para la sociedad y la productividad del país.

En este sentido, la propuesta de la ENS se basa en el sistema de pilares que ha promovido la OIT. El primer pilar sería solidario, universal, que asegure un ingreso vital de ciudadanía -o ingreso antipobreza- a toda la población adulta mayor (piso de protección social). Inicialmente se focalizará en la población adulta mayor más vulnerable, para después extenderlo progresivamente a toda la población mayor que no recibe pensión. Un segundo pilar es equivalente al régimen de prima media, que incluye la afiliación obligatoria a todos los trabajadores/as con cotizaciones hasta 4 salarios mínimos legales, administrado por Colpensiones. Este pilar incluiría al 94\% de los trabajadores asalariados, y les aseguraría ingresos que no conlleven la disminución dramática de los ingresos, como les ocurre hoy a los pensionados de los fondos privados. Un tercer pilar será para trabajadores que puedan cotizar por encima de 4 salarios mínimos mensuales, a través de la apertura de cuentas de ahorro individual, de libre elección entre Colpensiones o los fondos privados, que permitan un ingreso adicional y superior a la pensión que le asegura el segundo pilar.

La crisis generalizada del sistema de protección social en Colombia demanda una reforma pensional integral que asegure la sostenibilidad del sistema, cuyo foco sea asegurar ingresos antipobreza a toda la población adulta mayor, equivalente a medio salario mínimo legal, esto como un derecho de ciudadanía y no como simple programa asistencial. La propuesta incluye dos puntos adicionales. Uno es la garantía de pensión mínima para todos los trabajadores que cumplieron edad y cuentan hasta con el $70 \%$ de semanas cotizadas, para ello se les seguirá descontando la respectiva cotización. 
El segundo es el restablecimiento del derecho a la negociación colectiva en materia pensional, acatando plenamente las recomendaciones del Comité de Libertad Sindical de Organización Internacional del Trabajo (OIT).

\subsection{Gobierno nacional: DNP, Ministerio del Trabajo, Ministerio de Hacienda y Presidencia de la República}

\subsubsection{Departamento Nacional de Planeación (DNP, autores: Santamaria y Piraquive)}

Las propuestas para reformar de nuevo el sistema general de pensiones en Colombia deben tratar:

- Equidad: "Los Beneficios deben ser iguales cuando los esfuerzos son iguales".

- Cobertura: "Aumento progresivo de todos los trabajadores al sistema para que estos reciban los beneficios en proporción a sus aportes".

- Solidaridad: "Es necesario que los aportantes y beneficiarios del sistema con mayor capacidad de pago efectúen contribuciones adicionales para financiar las prestaciones de los trabajadores y beneficiarios de menores ingresos".

- Sostenibilidad: "Los aportes y las prestaciones ofrecidas deben ser proporcionales para que el sistema sea sostenible".

- Ajustes al actual sistema de prima media: los ajustes al sistema están asociados a equilibrar la tasa de reemplazo de las pensiones que se obtienen en el RPM con relación a las que se vienen obteniendo en el RAIS y, en segundo lugar, elevar la edad para obtener el beneficio pensional a 65 años para los hombres, y a 60 años para las mujeres. Se requeriría que el IBL sea de toda la vida laboral y no solamente de los últimos diez años.

- Escenario de marchitamiento del régimen de prima media: este es un escenario en el cual se cancelan las afiliaciones al RPM, de tal manera que solo se quedan quienes están afiliados hasta la fecha. Ello implicaría que se mantendría la carga actual solamente con las cotizaciones de los afiliados que existan a partir del cierre del sistema.

Desde el punto de vista de equidad, al eliminar el RPM se eliminan los altos subsidios que perciben quienes se pensionan en dicho régimen (DNP, 2013, p. 51). El marchitamiento del RPM contribuiría a hacer más sostenible el sistema de pensiones, gracias a que los beneficios se obtienen en función de los aportes de los afiliados. Los subsidios a cargo del Estado se reducirían enormemente y harían más transparentes las cuentas del Gobierno. Sin embargo, en el corto plazo, las cuentas fiscales del GNC se verían seriamente afectadas, porque dejarían de recibir aportes entre el 0,3\% y el 0,6\% del PIB por cuenta del flujo de cotizaciones de los nuevos afiliados al RPM, y los traslados (DNP, 2013, p. 52).

- Alternativa de pilares mixtos: en este sistema, al contrario del que existe en la actualidad en el cual el RAIS compite con el RPM, se complementa. El sistema consiste en que los afiliados cotizan hasta un salario mínimo en el primer pilar, el cual es de prima media, y a partir de un salario mínimo cotizan en el RAIS. Estos dos pilares (uno público y el otro privado) se complementan con los BEPS y los subsidios del FSP.

\subsubsection{Ministerio de Hacienda y Ministerio del Trabajo}

- Ministerio de Trabajo: la Ministra de Trabajo, Alicia Arango, comentó que "la propuesta de reforma a la vejez del gobierno Duque tiene cuatro puntos clave: la edad para la pensión no se va a aumentar, los derechos adquiridos se van a respetar, la pensión sustitutiva sigue en firme y se revisarán las altas pensiones" (RCN radio, enero 16 de 2019). Y agregó, "No es solamente el punto de si hay suficiente cotización, hay una cantidad de temas como la formalización, la movilidad laboral que tiene que ver con la edad". La idea es que todos los trabajadores formales e informales, puedan contar con un ingreso al llegar a la edad de jubilación o retiro, bien sea mediante una pensión, o un apoyo del Estado. ¿Por qué el gobierno pretende conservar la edad vigente? Con la idea de que al retirarse el trabajador a esa edad, permite generar otros empleos y en condiciones más favorables para el sistema pensional.

- Ministerio de Hacienda: el Ministro de Hacienda, Alberto Carrasquilla, en su intervención en el XII congreso (Cartagena, abril 5 de 2019) de la Federación Internacional de Administradores de Fondos de Pensiones (Fiap) y de Asofondos, expresó que, "En Colombia no habrá reforma pensional, sino reforma a la protección de la vejez", pues, "la nueva perspectiva amplía el marco del debate más allá del 20\% de la población 
que hasta ahora ha concentrado las discusiones sobre jubilación en el país, considerando que el otro $80 \%$ de la población vieja (en edad de estar jubilada) en Colombia está distribuido así: $34 \%$ no tiene ningún tipo de ahorro y otro $46 \%$ que, aunque ha ahorrado, no tiene suficiente capital para pensionarse". Para atender a ese amplio segmento poblacional, hasta ahora desprotegido, el ministro Carrasquilla informó que en marzo de 2019 comenzó a sesionar la Comisión de Reforma de Protección a la Vejez.

La "Comisión de Reforma de Protección a la Vejez" está integrada por el Ministerio del Trabajo, Ministerio de Hacienda y Crédito Público, Departamento Nacional de Planeación, Superintendencia Financiera, Unidad de Regulación Financiera y Colpensiones. Apoyan el proceso como expositores de las audiencias a la Comisión, el BID, Banco Mundial, Mercer, Unidad de Gestión Pensional y Parafiscales, Fedesarrollo, Fasecolda, Bolsa de valores de Colombia, Asofondos, ANIF, Universidad de los Andes, Universidad Javeriana, Central Unitaria de Trabajadores y Confederación de Pensionados.

\subsubsection{Banco de la República}

La propuesta de López y Sarmiento (2019) comparte la fórmula propuesta por Fedesarrollo (Leonardo Villar), de adoptar un sistema multipilar que integre en un primer pilar de ahorro al régimen de prima media, en un segundo pilar el ahorro individual, $\mathrm{y}$ un tercer pilar al régimen no contributivo de Colombia Mayor y los BEPS. Esta propuesta implica que en el régimen de prima media solo se asegure el primer salario mínimo para los que alcancen los requisitos para pensionarse, y el resto de la pensión estaría a cargo del régimen de ahorro individual.

La propuesta incluye un aumento en la edad de pensión para los actuales trabajadores que les faltan 20 años o más para pensionarse. En afinidad con el Gobierno, los autores enfatizan el respeto por los derechos adquiridos para quienes les faltan menos de 20 años para pensionarse.

La propuesta también contempla un aumento en la tasa de cotización de 16 a 18 por ciento sobre los salarios, y una disminución de las semanas de cotización para el RPM de 1.300 a 1.150 del RAIS. Esta reducción iría acompañada del incentivo al ahorro voluntario para cotizar más semanas y aumentar de esta forma la tasa de reemplazo de todos los cotizantes del sistema multipilar.

El sistema multipilar descarta la pretensión de acabar con alguno de los dos regímenes, lo que consideran inconveniente desde todo punto de vista, pues eliminar o marchitar el régimen de prima media generaría a mediano plazo un mayor déficit para el Gobierno a tener que financiar a Colpensiones para el pago de las pensiones vigentes, sin el recaudo de aportes por nuevos afiliados.

\section{Propuesta del autor}

ANIF, Fedesarrollo y DNP, coinciden en proponer que Colpensiones asuma los aportes de todos los trabajadores hasta de un SMMLV, y se encargue de otorgar el primer tramo de la prestación pensional, el cual garantizaría una pensión de igual monto. A partir del salario mínimo, las cotizaciones en calidad de ahorro o capitalización individual sean administradas por los Fondos de Pensiones Privados (RAIS).

La propuesta de la Sindical Obrera (CTC y CUT) se basa en el sistema de pilares promovido por la OIT. El primer pilar es solidario, universal, que asegure un ingreso vital -o ingreso antipobreza- a toda la población adulta mayor (piso de protección social). Un segundo pilar, equivalente al régimen de prima media, que incluye la afiliación obligatoria a todos los trabajadores/as con cotizaciones de hasta 4 salarios mínimos legales, administrado por Colpensiones. Un tercer pilar para trabajadores que puedan cotizar por encima de 4 salarios mínimos mensuales, a través de la apertura de cuentas de ahorro individual, de libre elección entre Colpensiones o los fondos privados, complementario al pilar 2.

La propuesta de Asofondos es más agresiva, desaparecer el RPM y a Colpensiones y liberar al sector público de su participación en el régimen contributivo, para que asuma el régimen no contributivo (BEPS y Adulto Mayor).

Los ejercicios financieros arrojan como resultado que los aportes y los rendimientos acumulados por cotizaciones de hasta un SMMLV con la restricción constitucional de una pensión mínima igual, genera pérdidas a los fondos privados. Actualmente a los cotizantes que no alcancen un ahorro más los rendimientos acumulados superior a los $\$ 190$ millones (a precios de 2019), quedan excluidos de recibir una pensión de un SMMLV en el RAIS, y su ahorro más los rendimientos acumulados les son devueltos. 
A continuación, se presenta una propuesta con tres escenarios alternativos o complementarios:

\subsection{Escenario 1: Mantener el sistema actual de los dos regimenes (RPM y RAIS) y fortalecer el RPM}

- Ampliar la cotización del 16\% al 18\% (12\% empleadores y $6 \%$ empleados).

- Aumentar la edad de pensión: hombres a 65 y mujeres a 63 años (de hecho, la edad de retiro forzoso de los empleados públicos es de 65 años).

- Incrementar el número de semanas cotizadas de 1.300 (cerca de 25 años) a 1.450 semanas (entre 28 y 29 años de vida laboral) como requisito para tener derecho a la pensión. El promedio del disfrute de la pensión se reduciría de 19 a 16 años.

- Continuar con el promedio de salarios de los 10 últimos años (IBL) para establecer la base y el monto o tasa de reemplazo de la pensión, reducir el monto de $80 \%$ a $75 \%$ y la base del $65 \%$ al $60 \%$ con un $2 \%$ adicional por cada 50 semanas adicionales cotizadas.

- Establecer un impuesto del $2 \%$ a las pensiones superiores 10 SMMLV del RPM para la sostenibilidad del mismo.

- Los afiliados que a la edad de pensión (hombres 65 y mujeres 63) no hayan podido alcanzar el número de semanas requeridas (1.450) recibirán un porcentaje inferior al $2 \%$ por cada 50 semanas menos cotizadas hasta un mínimo de 500 semanas (este porcentaje inferior incluye a los cotizantes de un SMMLV). Para los afiliados con menos de 500 semanas cotizadas, transferir sus cotizaciones al régimen no contributivo del Adulto Mayor (escenario 3). Ejemplo: un afiliado que aporte sobre un SMMLV (\$828.116 en 2019), partiendo del tope de 1.450 semanas, si aportó 1.000 semanas tendría un porcentaje del $82 \%$ de pensión del mismo, y el de 500 semanas cotizadas obtendría el $62 \%$ de pensión del salario mínimo, y así sucesivamente para los aportes superiores a un SMMLV, para quienes el porcentaje comienza a descontarse a partir de la base del $60 \%$ del IBL.

El Instituto de Métrica y Evaluación de la Salud (IHME) de la Universidad de Washington, presentó en The Lancet, una de las revistas médicas, un estudio de 38 investigadores, quienes analizaron cientos de datos de 195 países, incluida Colombia.
Las estimaciones de los autores indican que, en el caso colombiano, la población pasará de tener una esperanza de vida promedio de 78,2 años, a una de 81 años en el 2040 (El Tiempo, octubre 17 de 2018).

\subsection{Escenario 2: eliminar la competencia entre RPM y RAIS}

- Afiliación obligatoria para todos los trabajadores, y los cotizantes de hasta cinco SMMLV sean administrados por Colpensiones bajo el RPM, y las cotizaciones (aportes) con sueldos superiores a este monto los administren los Fondos Privados bajo el RAIS, más los ahorros voluntarios que puedan hacer los cotizantes.

\subsection{Escenario 3: financiación del régimen no contributivo}

La deuda social en Colombia es histórica, la pobreza y la pobreza extrema están alcanzando cifras cercanas al sesenta por ciento de la población, lo cual requiere la contribución de todas las organizaciones empresariales y sociales sin excepción (contribuyentes o no del impuesto de renta), y de todos los declarantes del impuesto a la renta, para superar los problemas de la pobreza y la pobreza extrema (de hecho, las organizaciones empresariales y sociales, y algunos contribuyentes alcanzan sus beneficios y los podrían optimizar con un mejor entorno económico, social, ambiental y de seguridad).

Los Adultos Mayores (65 años) en pobreza o en pobreza extrema, desprotegidos, recibirán una pensión del Estado no inferior al 50\% (\$414.058) de un SMMLV (\$828.116 en 2019), la cual se puede complementar con aportes a los BEPS. Una persona con ingresos en 2018 por debajo de $\$ 257.433$ se considera en pobreza monetaria, y por debajo de $\$ 117.605$ en pobreza extrema. Se trata entonces de complementar ese ingreso hasta el 50\% del SMMLV, así: la persona en pobreza extrema recibiría $\$ 296.453$ (\$414.058 - \$117.605) y la persona en pobreza monetaria $\$ 156.625$.

La pensión del Adulto Mayor sería financiada con el 1,0\% adicional sobre elvalor quele corresponda pagar por el impuesto de renta de los contribuyentes descritos, sin excepción. Adicionalmente, el GNC y los gobiernos departamentales y municipales, contribuirían con el 1\% de los ingresos corrientes (tributarios y no tributarios). Este impuesto sería administrado por Colpensiones. 
Nota: según "Estadísticas de Ingresos Tributarios Administrados por la DIAN 1970-2019”, el recaudo total del impuesto a la renta en el año 2018 fue de $\$ 68$ billones. Los ingresos corrientes del GNC son de $\$ 144,4$ billones, mientras el total de ingresos corrientes de los entes territoriales alcanzó los \$106 billones, para un total aproximado de $\$ 318,4$ billones. El 1\% equivaldría a $\$ 3,184$ billones para atender una población mayor de 65 años del régimen no contributivo aproximada de 2.854.490 personas (según resultados del censo de población y vivienda del DANE 2018, la población mayor de 65 años es de 4.391.523, y de esta cifra, el $65 \%$ corresponde a personas sin protección social).

\section{Referencias}

Administradora Colombiana de Pensiones, Colpensiones (2018). Informe de Gestión al Congreso de la República. Bogotá, marzo.

Aumento de esperanza de vida en Colombia (octubre 17 de 2018). El Tiempo. Recuperado de https:// www.eltiempo.com/salud/esperanza-de-vidaen-colombia-para-el-2040

Arrieta, C. (2011). Las reformas del sistema pensional colombiano. Colombia: Friedrich Ebert Stiftung Colombia (FESCOL).

Apella, I., Rofman, R. y Vezza, E. (2013). Más allá de las Pensiones Contributivas. Catorce experiencias en América Latina. Buenos Aires: Banco Mundial.

Clavijo, S. (2017). Elementos para una reforma estructural pensional. Bogotá: ANIF.

Berstein, S., Bosch, M., Castellani, F., Oliverim, M.L. y Villa, J.M. (2015). Diagnóstico del Sistema Previsional Colombiano y Opciones de Reforma (Nota Técnica No. 115). Banco Interamericano de Desarrollo (BID), Bogotá.

Cedetrabajo. (s.f.). La reforma pensional: fortalecer el capital financiero y pauperizar a los trabajadores. Bogotá: Cedetrabajo.

Comisión del Gasto Público. (2018). Informe Ejecutivo. Bogotá: Ministerio de Hacienda.

Contraloría General de la República. (2018). La reforma pensional, controversia analítica. Revista de Economía Colombiana, (352).

Jorgensen, S. L. (1999). Social protection as social risk management: Conceptual Underpinnings for the Social Protection. Sector Strategy Paper, Social Protection 38 (Discussion Paper No. 9904). Washington, D.C.: Banco Mundial.

Holzmann, R. y Jorgensen, S. L. (2000). Social Risk Management: A New Conceptual Framework for Social Protection and Beyond. Social Protection
Discussion (Paper No. 0006). Washington, D.C.: Banco Mundial.

La "bomba social a punto de estallar" (marzo 05 de 2018). El Nuevo Siglo. Recuperado de http:/ /www. Las pensiones en Colombia "bomba social a punto de estallar".

La propuesta de reforma a la vejez del gobierno Duque (enero 16 de 2019). RCN radio. Recuperado de http:/ /www. La reforma a la vejez del gobierno Duque. O, ver también: http:/ /www. La reforma a la vejez del gobierno Duque

López, M. y Sarmiento, E. (2019). El sistema pensional en Colombia. Borradores de Economía, No. 1078. Bogotá: Banco de la República.

Montenegro, S. (2018). Propuesta de reforma integral para la vejez. Asofondos en Seminario Internacional. Bogotá: Documentos, Banco de la República.

Necesidad urgente de realizar una reforma pensional de fondo (marzo 22 de 2019). Recuperado de http:/ /www. Reforma pensional urgente.

OCDE. (2015). Reformar el sistema pensional y los subsidios para personas de la tercera edad. En Estudios económicos de la OCDE Colombia. Bogotá: OCDE (pp. 37-42).

Propuesta de ANIF a la "Comisión de Protección a la Vejez" (junio 10 de 2019). Portafolio. Recuperado de https://www.portafolio.coreconomiaranifratifica-su-propuesta-de-reforma-pensionalestructural.

Santa María, M. y Piraquive, G. (2013). Evolución y alternativas del sistema pensional en Colombia (Documento 398). Dirección de Estudios Económicos, Departamento Nacional de Planeación (DNP),

Villar, L. y Forero, D. (2018). Elementos para una propuesta de reforma del sistema de protección económica para la vejez en Colombia. Cuadernos Fedesarrollo, (58). 